\title{
Estimating and exploiting the capacity of urban street networks
}

\author{
CHIARA COLOMBARONI, NATALIA ISAENKO \\ Department of Civil, Constructional and Environmental Engineering (DICEA) \\ Sapienza University of Rome \\ Via Eudossiana, 18 - 00184 Rome
}

ITALY

\begin{abstract}
The paper deals with the problem of estimating and exploiting traffic capacity of different road elements (link, nodes, network) and presents the results obtained by performing a systematic investigation of the role that the parameters of a microscopic simulation model play on the macroscopic representation of different road elements. An analysis of traffic parameters has been performed using a microsimulation software package to identify the most important parameters affecting the arterial capacity and to calibrate driver's behavior models through macroscopic traffic observations.
\end{abstract}

Keywords: Road Traffic, Traffic Control, Capacity, Performances, Micro-simulation

Received: March 9, 2020. Revised: September 3, 2020. Accepted: September 20, 2020. Published: October 8, 2020.

\section{Introduction}

Exploiting the actual capacity of urban traffic networks should be the first goal in order to relieve the heavy congestion that usually affects large urban areas.

Anyway, maximizing the traffic network capacity is a rather complex problem and even estimating the actual capacity of the network is not a simple task. While the capacity of a road link can be easily determined by measuring the traffic outputs, the capacity of a node is a little more difficult problem, since the total traffic served depends on its distribution on the different approaches as well as on the traffic control rules: yield or stop signs, roundabout, pre-timed or traffic-responsive control. Specifically, even if signals can improve the road junction capacity with respect to unsignalized junctions, they are usually designed and set to minimize the average delay. Smith [1] highlighted how traffic-responsive signals that are timed according to the equisaturation criterion can lead to unstable solutions and can then worsen the current conditions. They also introduced a capacitymaximizing policy that ensures traffic stability.

The problem is even more complicated in the road networks because of the effects of drivers' route choice. Numerous studies on the joint problem of traffic signal settings and traffic assignment have been undertaken either to deal with the optimization of the network performance or to describe the mutual interaction between traffic-responsive signals and drivers' route choice adjustments [2]. Although the average individual travel time is usually taken as the objective function of the global optimization problem, useful information about the efficiency of the signal control in exploiting the network capacity was provided by the study of the non constrained problem of optimizing traffic routes and signal settings jointly [3].

It is important to notice that steady-state network models based on the notion of traffic equilibrium fail to catch relevant dynamic aspects like queue progression and absorption, which affect the capacity of single network elements: queues can saturate the link storage capacity and reduce the capacity of upstream nodes and even of crossing links [4].

Under certain critical conditions, spillback queues coming from different links join each other and give rise to gridlock. This is a critical phenomenon for traffic network simulation models since the traffic flow on these links stops absolutely [5].

Colombaroni et al. [6] developed a model to estimate travel time on an expressway in a queue condition due to a capacity reduction, as in the case of an accident occurrence.

Torrisi et al. [7] derived the road network capacity for the urban network of the Italian town of Catania by using a dynamic traffic assignment model calibrated through Floating Cara Data and Radar measurements. Shepelev et al. [8] studied the intersection road capacity and developed a Fuzzy Model to estimate it.

Shi et al. [9] analyzed the effect of different features (scale, structure, demand pattern) of a grid network on its capacity using a Cellular Automata model.

Yang et al. [10] and later, Zhang et al. [11] studied the Equilibrium Network capacity problem and solved it as a maximization of trip distribution and assignment problem for a network as a whole. 
However, the goal of the present paper is to analyze the capacity of the single network elements depending on their internal features.

In order to allow microscopic simulation models reproducing road traffic in critical conditions adequately, it is very important to investigate factors and relationships that affect traffic stability and then capacity.

The aim of the paper is to discuss the basic factors that affect the capacity of single road elements (link, nodes, arteries) and the main problems arising from the use of new sophisticated microsimulation models in traffic control design. In particular, a sensitivity analysis of the most significant parameters of microscopic models is performed in order to provide useful indications on their use in traffic engineering. The second part of the paper deals with the problem of gridlock on a simulated network and illustrates it through a realistic example on a portion of the road network of Rome.

\section{Opportunities and warnings in using microscopic simulation models}

Microscopic models describe the drivers' behavior in a traffic stream and vehicle movements' interaction at an individual level. Thus, they are a powerful tool to get an in-depth analysis of traffic flow under various conditions, and they are more and more used by traffic engineers as design support to estimate the impacts of traffic control measures.

Of course, the need for increased accuracy in representing traffic phenomena also implies a greater complexity of the model. Indeed, the most advanced micro-simulation models are characterized by a complex modeling structure and very large numbers of parameters that make the calibration process and very long and burdensome. Calibration is crucial to avoid that the very sophisticated representation of traffic provided by micro-simulation models does not fit the real phenomenon. Only an accurate calibration ensures to achieve an accurate representation of real traffic phenomena.

Correct calibration of microscopic models would require intensive experiments concerning the behavior of a significant sample of drivers in order to estimate the numerous coefficients of the different models that best fit the drivers' behavior in different driving choices: car following, gap acceptance, emergency braking, lane changing, etc. The task is huge, provided that one of the most advanced microsimulation models, TransModeler, has about 40 different types of parameters that describe drivers, road, and vehicle fleet [12]. Moreover, many parameters are disaggregated with respect to driver and vehicle classes; some of them are expressed as functions of other variables so that more coefficients are introduced. Next Generation Simulation (NGSIM) is a unique public-private partnership between FHWA and commercial microsimulation software developers, the academic research community, and the traffic microsimulation community. The NGSIM initiative carried out a vast work of calibrating of complex models performing large experiments. Even if the initiative is appropriate and very useful from both a theoretical and a methodological point of view, the results obtained can not be easily extrapolated from one country to another without a sound validation on real data. As a matter of fact, applying an even very sophisticated microsimulation model by using default parameters may lead to very unrealistic results.

Of course, replicating intensive experiments on driver behavior is not feasible in each specific area to simulate. The simplest practical approach consists of acting on some parameters in order to fit the capacity of the actual bottlenecks of the network and reproduce so the observed traffic flow. However, as the number of parameters is very large, it is not immediate to individuate, which are the most appropriate to change.

In this regard, a sensibility analysis of capacity with respect to the most relevant parameters may be useful theoretically and help traffic engineers in reducing and simplifying their effort for calibrating microsimulation models.

\section{Micro-simulation software}

The analysis of traffic parameters has been performed by using the above-mentioned microsimulation software package Transmodeler.

Different single elements of the road network (links, nodes, arteries) have been simulated individually under different traffic demand scenarios. For each demand condition, one parameter at a time has been varied within a given reasonable range of variables, and the corresponding values of outflow and speed have been recorded as measures of capacity and performances of that element.

Several parameters have been set as constant. This is the case of geometric and mechanical characteristics of the car fleet, which have been set to describe the average vehicle fleet distribution in Italy.

Mass, length and power data are reported in Tab. 1. Diagrams of the maximum acceleration (or deceleration) as functions of the speed are shown in Fig. 1.

It is worth mentioning that mechanical characteristics are referred to the maximum vehicle performances. 
Other different values have to be set according to the road features, such as desired speed.
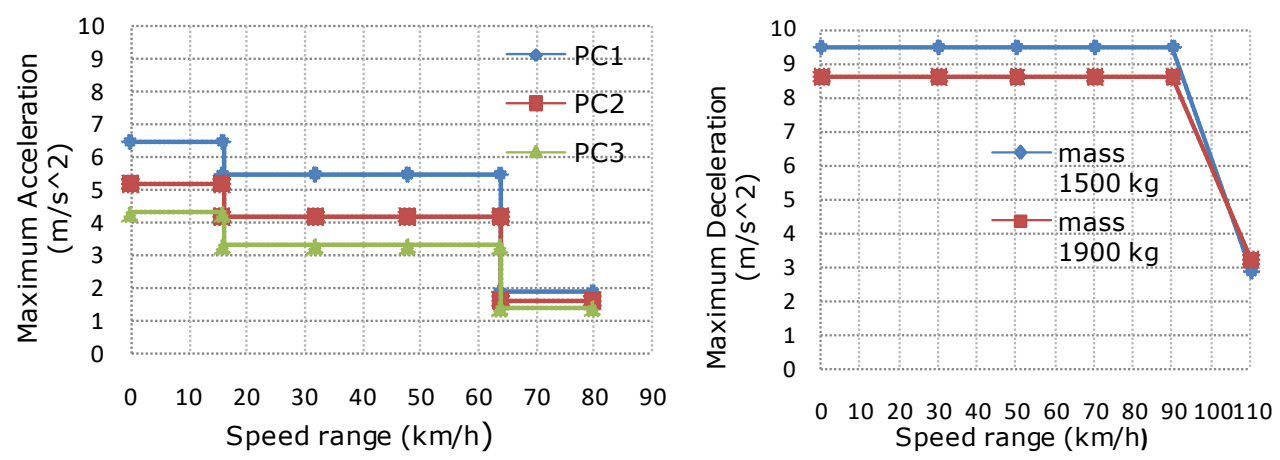

Fig. 1 Maximum acceleration and maximum deceleration as functions of the speed

Tab. 1 Vehicle fleet parameters

\begin{tabular}{lcccc}
\hline \multicolumn{1}{c}{ Vehicle class } & $\begin{array}{c}\text { Mean } \\
\text { Length }(\mathrm{m})\end{array}$ & $\begin{array}{c}\text { Power } \\
(\mathrm{kW})\end{array}$ & $\begin{array}{c}\text { Mass } \\
(\mathrm{kg})\end{array}$ & $\begin{array}{c}\text { Percentage of } \\
\text { fleet } \%\end{array}$ \\
\hline PC1 - High performance passenger cars & 4,5 & 125 & 1500 & 30 \\
PC2 - Middle performance passenger cars & 4,3 & 90 & 1280 & 30 \\
PC3 - Low performance passenger & 3,5 & 62 & 1175 & 20 \\
PU - Pickup trucks or utility vehicles & 5,2 & 130 & 1900 & 10 \\
M - Motorcycles & 2,4 & 80 & 160 & 10 \\
\hline
\end{tabular}

The operational values of acceleration, deceleration, and speed depend on both external conditions, that is, traffic congestion, and individual preferences, which are taken into account through statistical distributions.

\section{Link analysis}

While macroscopic models are continuum mathematical relations between some two or three global quantities that describe the traffic stream on a link as a whole (namely: flow, speed, density), microscopic models aim at reproducing the behavior of a generic driver within the traffic stream. On single-lane road segments, the foremost relevant form of interaction between drivers is the car following behavior. On multiple lane road segments, drivers have an additional degree of freedom, and another important factor is lane-changing behavior. The car-following model used in this analysis generalizes the so-called GHR model by introducing an asymmetric sensitivity function and a random term [13]:

$$
\frac{d v_{n+1}}{d t}(t+\tau)=\lambda^{ \pm}\left[v_{n}(t)-v_{n+1}(t)\right]+\varepsilon_{n+1}
$$

with the following parameters:

$\mathrm{v}_{\mathrm{n}}$ is the speed of the generic $\mathrm{n}$-th driver in the traffic stream

- $v_{n}+1$ is the speed of the $\mathrm{n}+1$ driver that follows the $\mathrm{n}$-th one

- $\tau$ is the driver's reaction time

- $\varepsilon$ is a vehicle-specific error term for car following regime

- $\lambda$ is the sensitivity function defined as:

$$
\lambda^{ \pm}=\alpha^{ \pm} \frac{\left[v_{n+1}(t)\right]^{\beta \pm}}{\left[s_{n+1}(t)\right]^{\gamma \pm}}
$$

$\alpha^{+}, \beta+$ and $\gamma^{+}$are coefficients related to the accelerating phase

$\alpha-, \beta-$ and $\gamma-$ are coefficients related to the decelerating.

As the simulation package TransModeler used in this analysis does not allow changing the reaction time, the analysis is focused on the other relevant 
variables, such as the sensitivity function and the minimum spacing between stopped vehicles.

It is a well-known result of the theory of traffic flow [14] that a space inverse sensitivity function in the car following model $(\beta+=\beta-=1 ; \gamma+=\gamma-=0)$ provides the macroscopic Greenberg's model [15] at the steady-state:

$$
q=\alpha \ln \left(\frac{k_{J}}{k}\right)
$$

where $k_{J}$ is the jam density.

In this case, the coefficient of sensitivity $\alpha$ coincides with the critical speed $\mathrm{v}_{\mathrm{c}}$, that is the speed corresponding to the maximum flow. It is straightforward deriving that the capacity

$q_{\max }=k_{j} \cdot v_{c} \cdot e^{-1}$ is proportional to the critical speed.

Thus, link analysis is focused on investigating the relevance of the sensitivity on the capacity in a simulation environment, where many other factors are introduced. The experiment consists of simulating a two-way $11 \mathrm{~km}$ long road segment under such a heavy demand that realizes a continuous traffic inflow. A bottleneck in the middle reduces the carriageway to only one lane so that the outflow identifies the one-lane link capacity.

Experimental results obtained in micro-simulation are consistent with the steady-state theory up to a critical value, after which the capacity becomes constant as the sensitivity coefficient $\alpha$ varies (Fig.2).

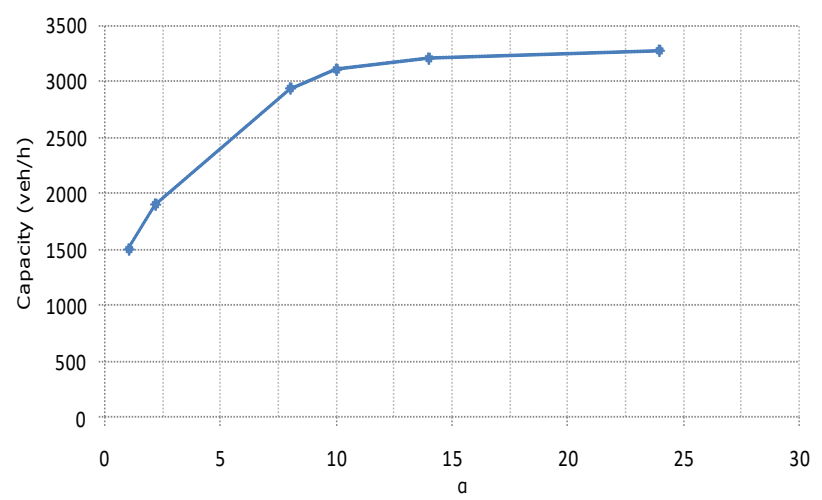

Fig. 2. Relationship between capacity and sensitivity. The experiment is repeated under many different values of the parameters of sensitivity and the minimum spacing between stopped vehicles.

This is due to the occurrence of incoming, more unstable conditions for larger values of sensitivity. In fact, ceteris paribus, the traffic system becomes unstable when the sensitivity is greater than $0.5 \tau^{-1}$ [14], and under unstable conditions, the deductions derived from the steady-state speed-density relationship do not hold.
It is interesting to analyze how the microscopic simulation model reproduces the macroscopic relationship between speed and density as the inflow varies. In this regard, a two-lane urban highway has been simulated for different values of inflow. The artery has a bottleneck at its end, in order to replicate the observed traffic conditions on the highway 'Tangenziale Est' in Rome, where real data were collected.

Fig. 3 highlights that the experimental values measured in different sections and time intervals during the simulation (left side) are consistent with the observed data as well as with the macroscopic model (right side) calibrated on these data.

\section{Node analysis}

Nodes are critical elements of the network since the same road space at junctions has to be used alternatively by conflicting traffic streams. The capacity of the junction is lower than that of a link having the same road characteristics (width, slope, etc.). Indeed, the junction has to be cleared by vehicles moving in one direction before the conflicting movements can cross it so that there is a lost time between the two conflicting traffic stream movements, which determines a loss of capacity with respect to monodirectional traffic, as it occurs on links.

The capacity of unsignalized junctions depends on the distribution of traffic on different approaches, other than on the priority rule.

Theoretically, the main road should be unaffected by traffic on the secondary one at junctions with yield or stop signs. Thus, the capacity of a priority intersection is usually seen as the maximum outflow of the minor road [16].

In the scientific literature, there are many statistical models [17] that express the capacity of the minor road as a function of the average flow on the major road, the critical gap, and the follow-up time.

The analysis of unsignalized junction in microsimulation environment have been carried out by varying the flow on the minor road up to individuate the maximum value of the total flow on both the major and minor road, that is the effective junction capacity. Several experiments have been performed for different values of the critical gap, as well as other factors that describe the impatience of the drivers. The result obtained for junctions with yield sign is trivial. The only interesting question concerns the value of the critical gap that corresponds to the observed outflow of the minor road. In real cases, however, it has been often observed that 
drivers on the minor road, after some waiting time, are affected by increasing impatience and tend to accept even shorter time gaps in the main traffic stream up to reverse the priority rule. This is a significant factor of disturbance for vehicles on the major road. They are induced to decelerate, and so the outflow is reduced consequently, as observed, among others, in [18].

In the micro-simulation environment, such a more realistic behavior is better represented by unsignalized junctions without yield signs, subject

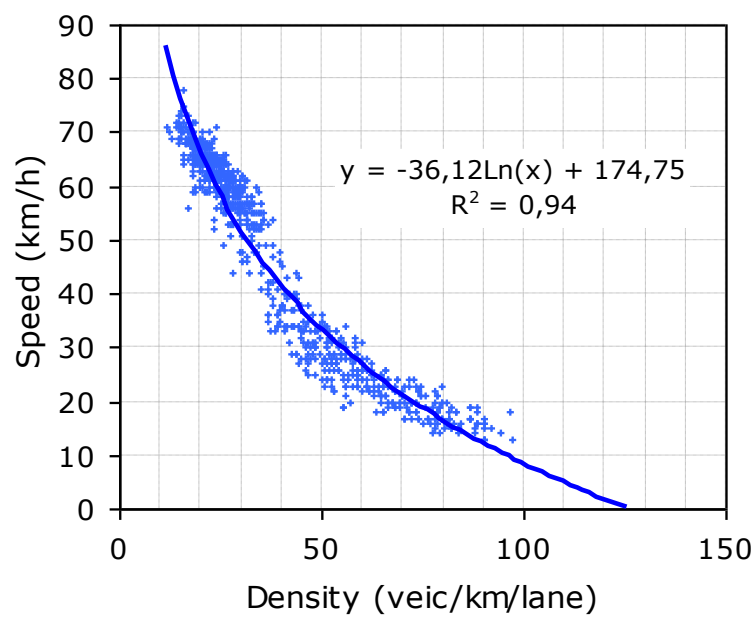

Fig. 3 Speed and density data collected on Tangenziale Est in Rome and the related calibrated model (on the left), simulated traffic states, and the related calibrated model (on the right).

The result of the simulation experiment is shown in Fig. 4. The outflow of the major road is constant up to a value of the inflow of the minor road, which corresponds to the junction capacity (for the given flow on the major road). Then, it decreases because of the priority reversal up to become equal to the flow of the minor road. Also, the total outflow decreases; of course, up to two times this value, higher values of the inflow on the minor road cannot be served, and the outflow on both is constant independently of the growing demand. Thus, as known, the maximum outflow exiting from unsignalized junctions depends on the distribution of the total inflow between the two approaches.

Capacity $c_{i}$ of an approach $i$ at a signalized junction is a straightforward consequence of the design variables (the cycle length $C$, and the effective green $\left.g_{i}\right)$, as well as of the saturation flow $s_{i}$ :

$$
c_{i}=s_{i} \frac{g_{i}}{C}
$$

It is well known that, as the inflow grows, also the cycle length has to be enlarged in order to reduce the incidence of the lost time and so increase the capacity.

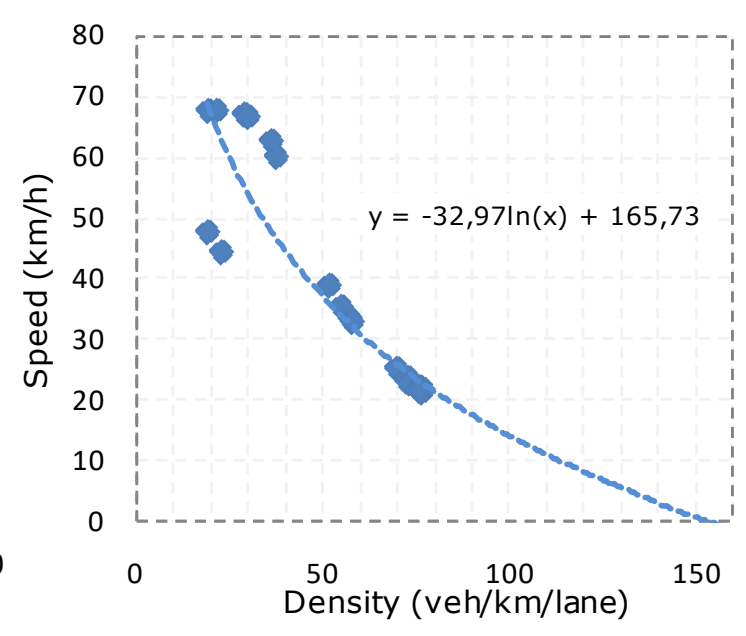

only to the right side priority rule. In the microsimulation, indeed, the critical gap is not such a relevant factor for the capacity of junctions without yield signs. The impatience factor becomes the most relevant.

Observations conducted on the field at an unsignalized intersection confirmed that the waiting time is the most relevant factor affecting the gap acceptance behavior [19].

Fig. 5 illustrates the capacity of a signalized junction as a function of the inflow on the secondary approach, assuming that the inflow on the major approach is given. Both approaches have one lane; each the green split is set according to the equisaturation criterion, and the cycle length is set according to either the minimum cycle or Webster's optimum.

When the cycle length is set at the minimum, the green time of each approach is the strictly necessary value to discharge the queue. Webster's criterion increases the green split in order to accommodate possible greater flows than the average. As the minor flow is increased, a progressively larger quota of the cycle length is assigned to serve it, so that the capacity of that approach increases and the other one is reduced. This is not the case of the minimum cycle since the flow on the first approach is kept constant; also, the green time to serve does not vary.

Both signal settings policies, of course, increase the total junction capacity as the flow on the second approach increases, since they both increase the cycle length. With respect to the minimum cycle, Webster's policy provides larger capacity. However, it is worth noticing that this should be properly seen as a 
potential capacity since the green times are set in order to accommodate possible greater flows than the average value. If the estimate of the average flow is correct and accurate, the actual outflow will be equal to the number of vehicle arrivals, that is, it will equal the capacity achieved with the minimum cycle length.

When the saturation flow of the two approaches is much different, it is convenient to increase the green split of the major road in order to increase the capacity junction. This is an obvious consequence of the definition of capacity. It is useful, noticing that it is also beneficial in terms of the average delays since it favors the approach that gives a greater contribution to the average value. Fig. 6 illustrates the relationship between average delay and green split ratio for a signalized junction, with asymmetric flow distribution.

It may be interesting to test the microscopic simulation model by comparing the average delay at an unsignalized junction with that obtained when introducing a signal. Fig. 7 refers to medium-heavy traffic conditions. Signalisation allows moderating the average delay when the traffic flow on the secondary road increases, provided that it does not exceed the capacity largely.

However, the average delay of the unsignalized junction becomes even six times higher than at a signal when the flow on the minor road grows up to $60 \%$ of that of the major road.

The key point is that the microscopic model with a simple priority rule (that is, without yield sign) predicts a complete priority reversal so that it assigns the greatest delay to the main road, which has the largest flow and then the longest queue, even if it has the right of priority. On the contrary, the priority model with yield or stop sign assumes unrealistically the main road is not at all affected by an even large inflow on the minor road. Thus, the microscopic simulation model used here is a suitable tool to estimate the actual capacity of critical junctions (which is a crucial issue in network simulation) but can not be seen as an accurate tool to estimate the average delay at each approach.

\section{Network analysis}

The capacity of the network is a rather general concept. Several empirical studies have been conducted in the ' 60 s on the total flow that can enter the city center in the unitary time period as a function of the area size and the total street surface [20].

As the junction capacity is affected by the traffic distribution between the approaches, the value of network capacity is also affected by the route choice behavior, which determines the distribution of inflow at the approaches of each junction. Drivers are assumed to be rational decision-makers, who aim at minimizing their own travel time.

Later studies [21] exploited computer simulation to investigate network-level relationships between the three fundamental variables of traffic flow, speed, flow, and density. They applied the two-fluid model and obtained a quite similar relationship to that holding for single facilities. A similar result has been obtained by the authors by using a dynamic traffic assignment model.

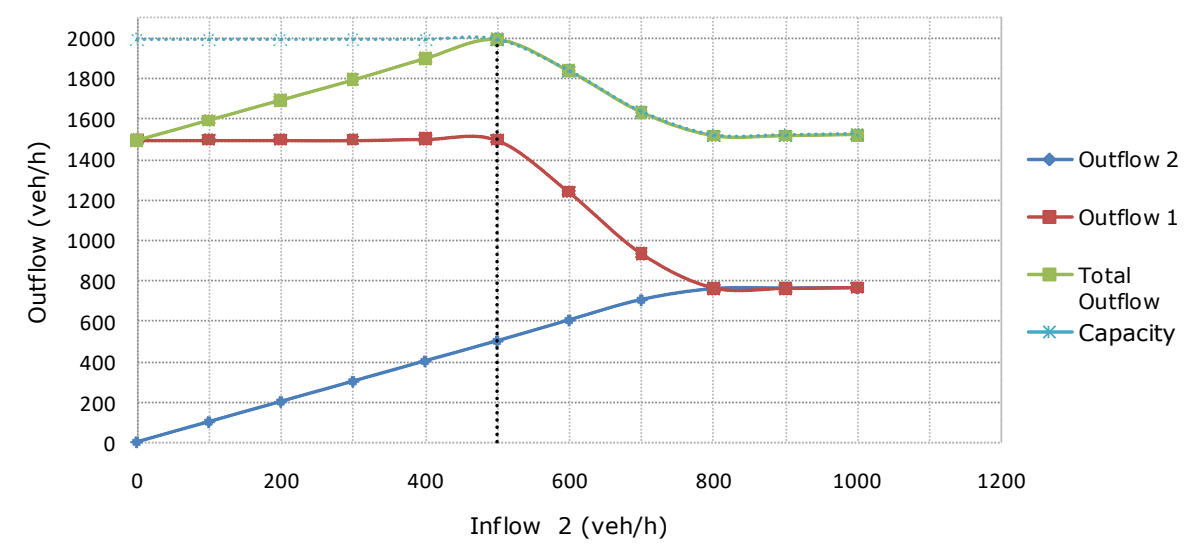

Fig. 4 Outflow at the approaches of an unisignalised junction and the junction capacity 


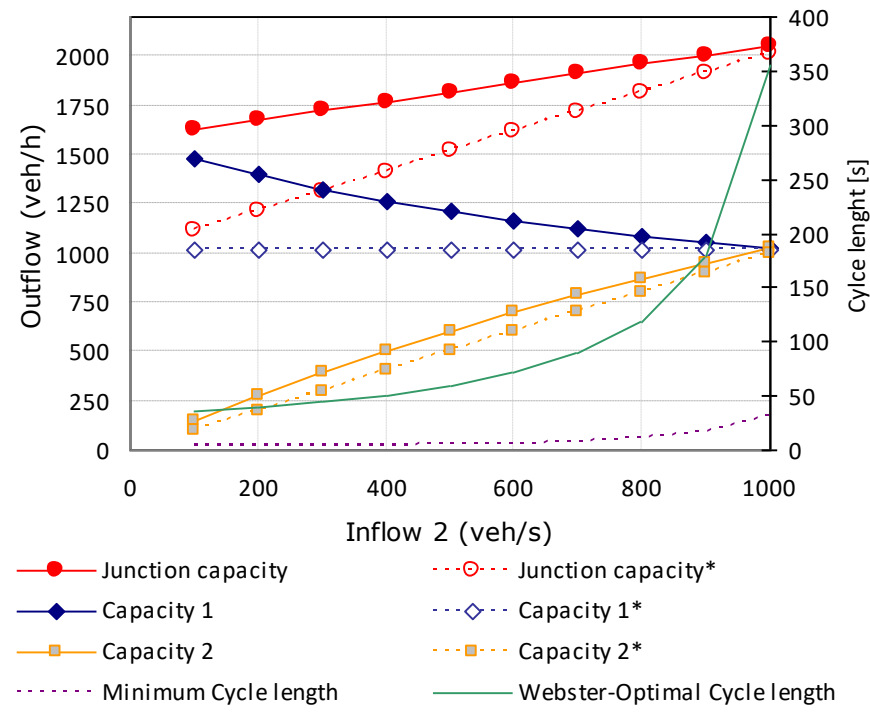

Fig. 5 Junction and approaches capacity at a signalized intersection corresponding to either minimum or Webster's optimal cycle length.

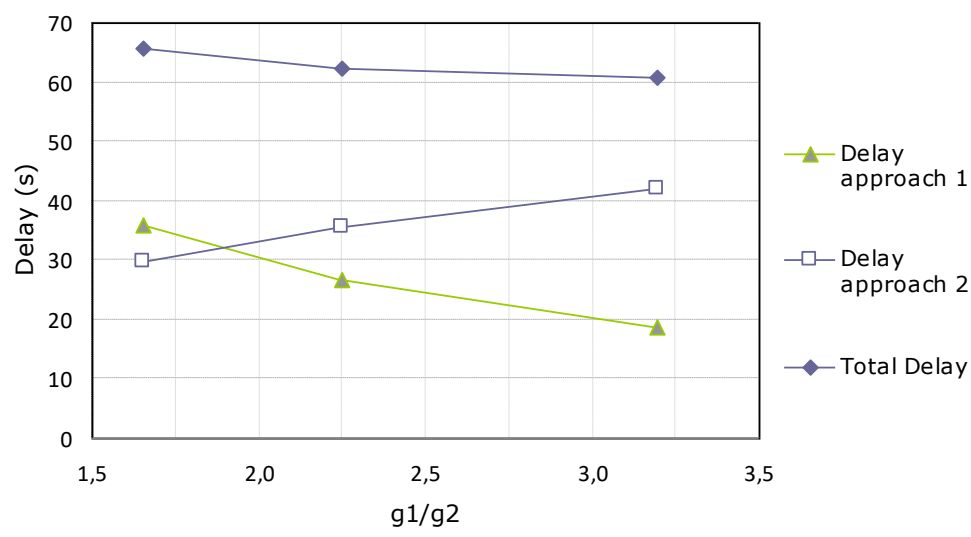

Fig. 6 Average unitary delay at approaches and at junction versus the green split ratio, where approach 1 has 4 lanes and an inflow of $6,000 \mathrm{veh} / \mathrm{h}$, and approach 2 has 1 lane and an inflow of $700 \mathrm{veh} / \mathrm{h}$.

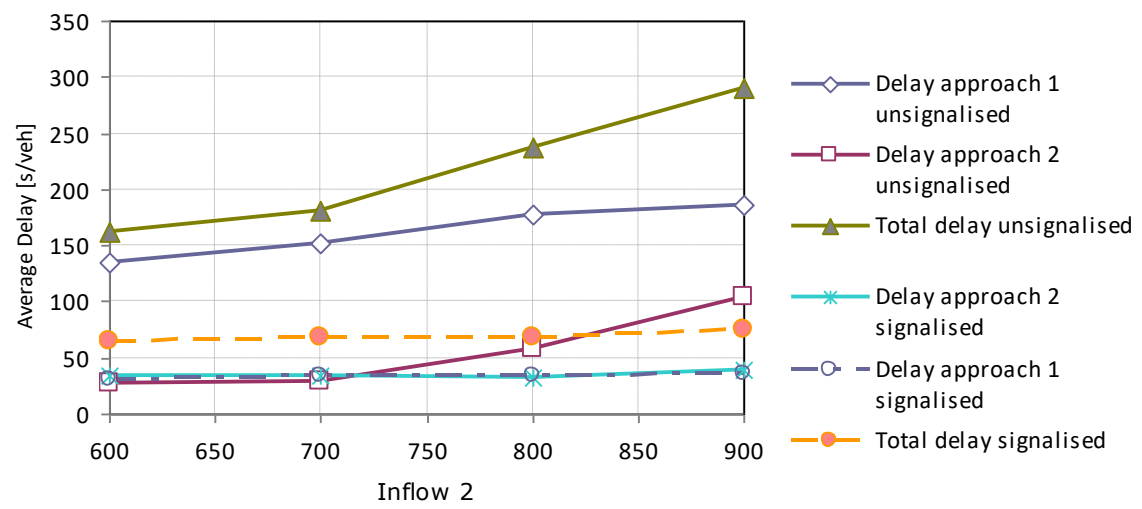

Fig. 7 Average unitary delay at the approaches and at a junction with and without a traffic signal. 
However, the capacity of a network does not depend only on the relationship between macroscopic flow and density. Another relevant factor in determining the network capacity is the link storage capacity.

As a matter of fact, when the storage capacity of a link is reached, queue spills back at upstream intersections and reduces their capacity. Moreover, the simulation of high congested networks by dynamic traffic models can often lead to gridlock conditions that block the simulation advancing.

In urban street networks, this is often the most critical condition, and it does not depend only on link flows, but on the queue length, that is on the combination of inflow, outflow, and link length.

Thus, in traffic network simulation, modeling of queue progression and clearance is crucial to ensure realistic and accurate simulation of the traffic congestion.

In several simulation analyses performed on realistic networks, it has been observed that gridlock occurs for values of link flow much smaller than the flow capacity.

Thus, it is expected that this is due to an excess of link storage capacity. In fact, a specific analysis of queue progression highlighted that the gridlock starts when a queue at one node blocks the traffic flow on a transversal link. The total block of traffic characterizing a gridlock condition occurs when all the links belonging to a circuit are saturated by a queue: in this case, dynamic network traffic models predict that the outflow is zero so that all links belonging to a circuit, each of them blocks the flow from the upstream link, and the queue can not be dissipated.

An example will be useful to explain the gridlock occurrence. It refers to a portion of the road network of Roma, simulated through the dynamic assignmentsimulation software Dynasmart [22].

To focus on a specific critical condition for gridlock starting, we analyze in detail the queue spilling back along with one of the main arteries in the direction of the city center (labeled as Via Nomentana, in Fig. 9) in the time interval between the 20th and the 80th minute of simulation.

Values of occupancy in Fig. 10 display how the queue starts to develop on the downstream link (identified by number 805 in the figure) around the 25th minute and then spills back to upstream links, reaching the last one (identified by number 714 in figure) at the 35th minute. After the 70th minute, all blocked vehicles on the downstream link start to move, and the queue is partially cleared at the end of the simulation.

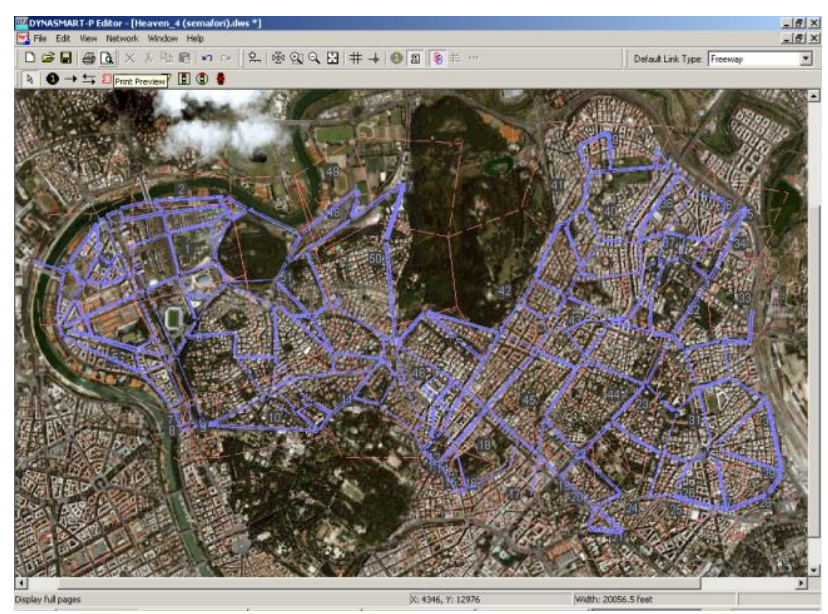

Fig. 8 Graph of the road network in Dynasmart.

Unlike in static traffic assignment, link saturation does not depend only on the flow entering the link, which is indeed only $25 \%$ of its saturation flow, but depends on the intersection at the end of the downstream link (left picture in Fig. 10). Spill-back and dissipation of queues across the network are displayed in Fig. 11. It highlights that the queue on the street 'via Nomentana' is induced by the queue on the transversal link labeled as 'Viale Gorizia', which blocks the outflow from 'via Nomentana' (picture on the left in the Figure).

Similar conditions occur on the parallel artery directed to the city center (street labeled 'Corso Trieste'), as well as on another transversal artery ('via di Santa Costanza'), which connects the former with 'via Nomentana'. Such a situation is critical for a gridlock condition. Anyway, in the example shown, the queue on 'Corso Trieste' does not spill back up to 'via di Santa Costanza' (picture in the middle of the figure), so that the gridlock condition is not attained, and the queue can be cleared.

The picture on the right in Fig. 11 also illustrates a definite gridlock condition occurring in the square labeled as 'Piazza Verbano', where the links that compose of the roundabout are not long enough to allocate the queues fed by the upstream roads. After the gridlock is reached, queues can not be cleared, and vehicles involved in it cannot reach their destination at the end of the simulation.

Accurate calibration of the most important parameters of the model, as link storage capacity and critical speed, is then required to properly simulate the usual recurrent congestion conditions. In such conditions, temporary over-saturation conditions occur, and even long and multi-branched queues spill back across the network but are always dissipated as the demand flow decreases. 


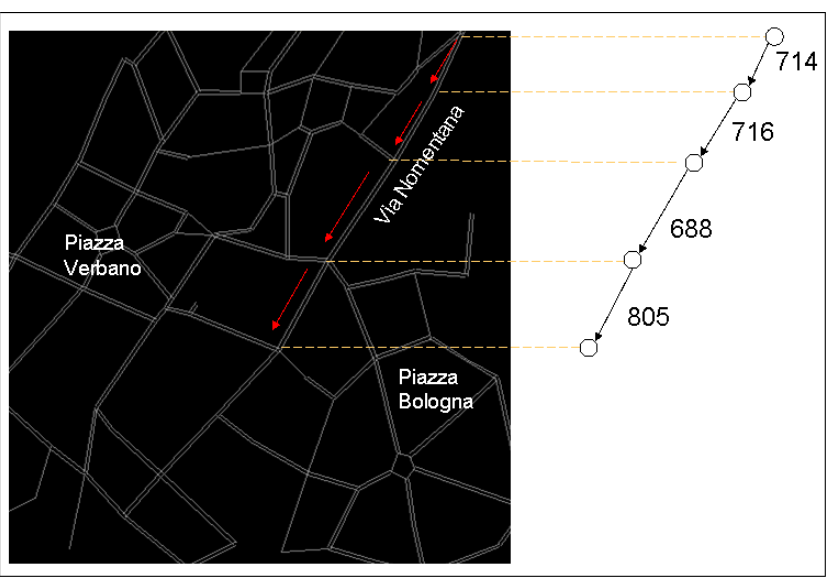

Fig.9 Links on the Nomentana street.

Actually, dynamic traffic models are conceived to simulate the congestion mechanism and can reproduce stopping and queuing phenomena at a bottleneck, but cannot reproduce the subsequent phase of traffic re-starting after the downstream bottleneck has been removed. This is true for all dynamic models, starting from the well-known carfollowing model that reproduces drivers' behavior along with a link, until the most advanced dynamic network models analyzed here.

To reproduce the phenomenon of slow queue movement and to allow outflow from saturated links, a minimum speed corresponding to jam density has been introduced in Dynasmart [23]. However, the simulation software Dynameq [24] applies a preventive gridlock algorithm that permits to stop the vehicles on a link when they are going to feed a potential gridlock situation.

\section{Validation of the microscopic model on a real case}

The microscopic model calibrated on the basis of the procedure explained above has been validated on real traffic data collected on a segment of a 4-lane 2way road urban artery in Roma, namely via Tiburtina, having 9 signalized junctions.

The vehicle fleet data used are summarised in Tab. 1 and Fig. 1. Behavioral parameters determined on the basis of observations of macroscopic variables and systematic analysis of the microscopic model are shown in Tab. 2.

Validation results show a good correspondence $(\mathrm{R} 2=0.86)$ between the output flow predicted by the model and the observed data (Fig. 12). Other than the satisfactory determination coefficient, it is worth noting that the slope of the regression line is very close to 1 , and this ensures that the model is unbiased.
Tab. 2: Behavioural parameters used in the microscopic simulation model

$$
\begin{array}{cc}
\text { Gaps between } & \text { Critical } \\
\text { stopped vehicles } & \text { Distance Car following parameters }
\end{array}
$$

Minimum Mean $\quad \begin{array}{llllll}\alpha^{+} & \alpha^{-} & \beta^{+} & \beta^{-} & \gamma^{+} & \gamma^{-}\end{array}$

$\begin{array}{lllllllll}0.3 \mathrm{~m} & 0.5 \mathrm{~m} & 31 \mathrm{~m} & 18 & 0.8 & -1 & -1 & 0 & 0\end{array}$

Fig. 13 shows a picture of the simulation of a complex junction along the artery. The junction revealed to be critical in the simulation process because of small links where queues of left-turning vehicles may be stored. The small storage capacity of links, in fact, may induce the gridlock phenomenon. However, a careful calibration of behavioral and physical parameters allowed simulating the observed queuing process quite realistically.

\section{Need for further analyses}

The paper illustrated a procedure for calibrating microscopic simulation models through a systematic correlation analysis between microscopic variables and observed macroscopic data. Although validation results are reasonable and statistically significant, a more sound and detailed calibration of microscopic models should include real experiments on the field, which are now facilitated by the existence of accurate Global Positioning System (PGS) sensors.

Experimental activities have been conducted to develop a car-following model based on the Neural Network paradigm using a fleet of few GPSequipped vehicles (Colombaroni et al. [25]). This research activity is now addressed to implement a deep learning algorithm to develop a new carfollowing model.

Further analyses will concern the use of the macroscopic dynamic traffic assignment model tackled by Fusco et al [26] in which the capacity constraints will be introduced.

For an accurate calibration of the model the Floating Cara Data potentials will be exploited by applying the spatial distributions of individual FCD positions method studied by Isaenko et al [27].

These considerations will find a huge applicability in the research field of Road Traffic Monitoring using new technologies like Vehicular Ad-Hoc Networks (VANETs) yet explored in De Felice et al. [28]. 

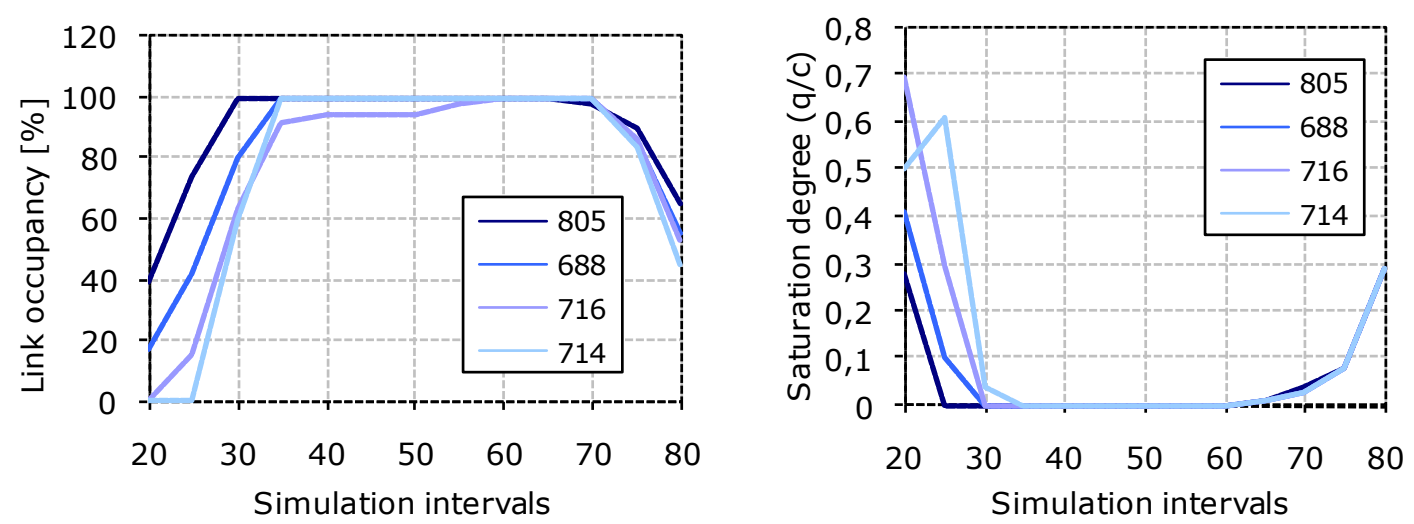

Fig. 10 Nomentana street links occupancy as computed by Dynasmart.
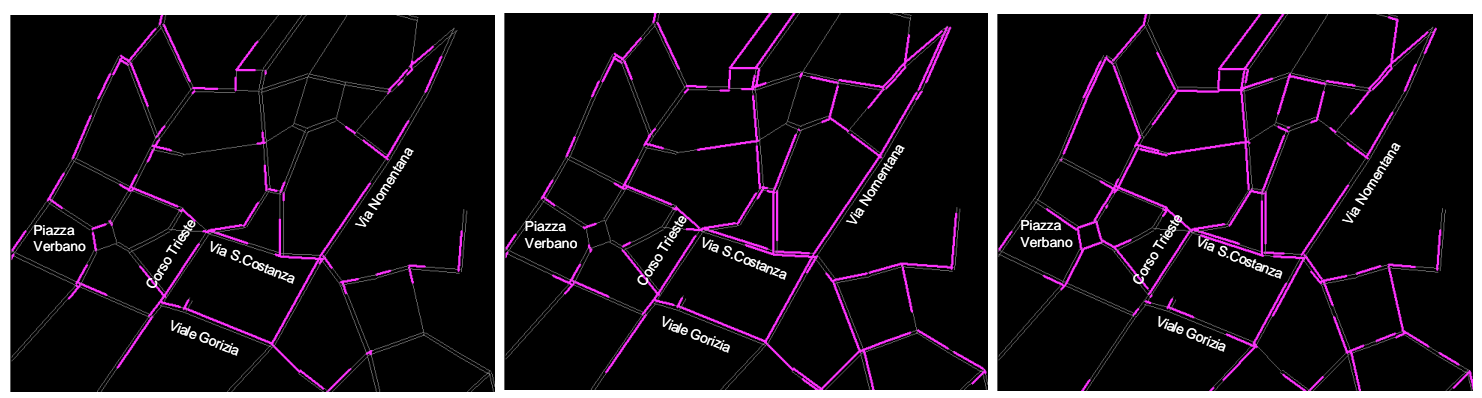

Fig. 11 Queue movement across the network as computed by Dynasmart in three different time instants.

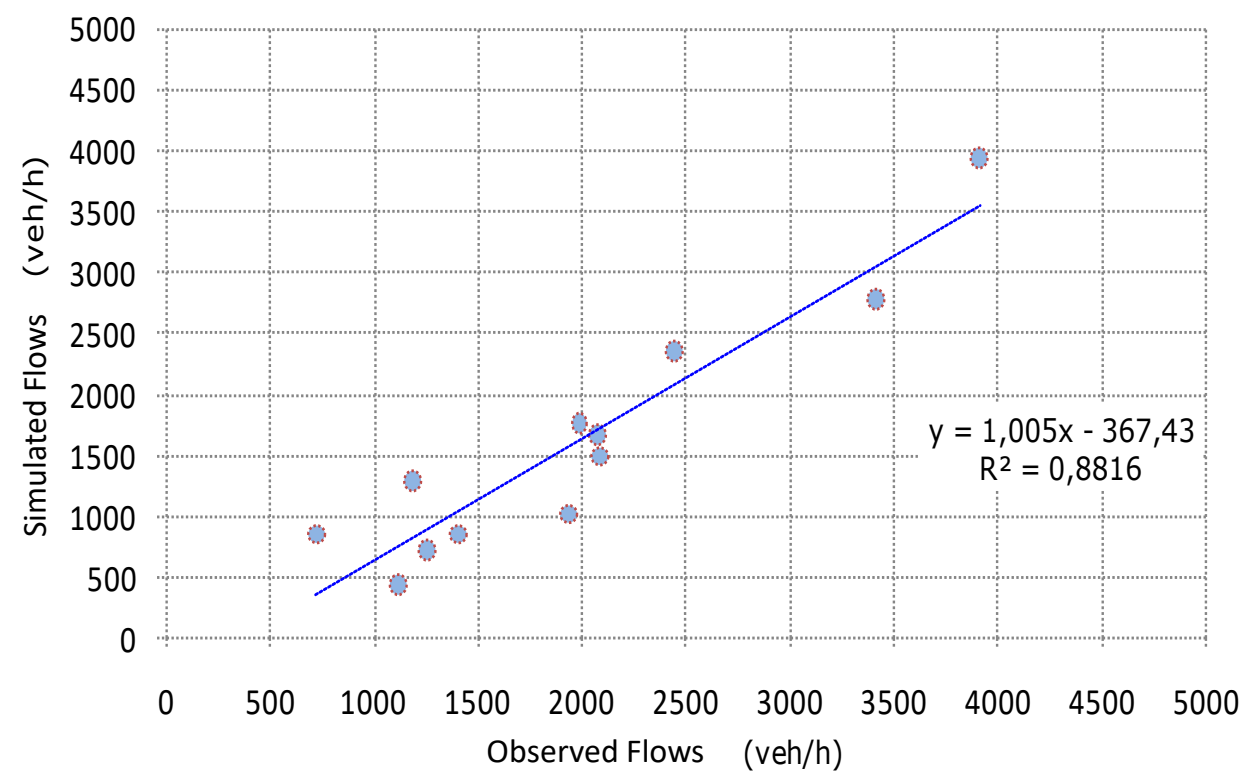

Fig. 12 Validation of the artery model of Via Tiburtina in Rome through observed traffic flows. 


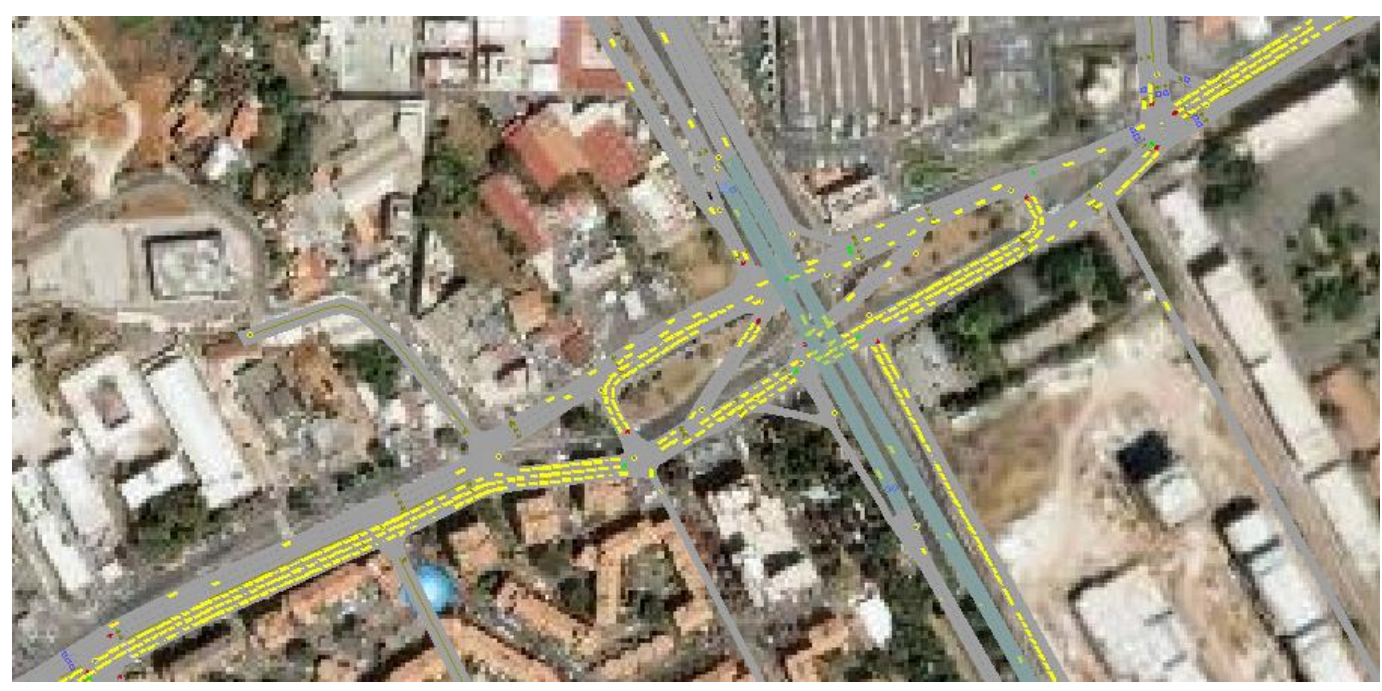

Fig. 13 Picture of the microscopic simulation showing spillback queues at a complex junction where gridlock condition may occur.

\section{Conclusions}

An analysis of traffic parameters has been performed by using a well-established microsimulation software package (Transmodeler) to identify the most important parameters that affect the arterial capacity and to calibrate driver's behavior models through macroscopic traffic observations.

The analysis highlighted the foremost calibration problems:

- a large number of parameters to calibrate for the different models (car following, gap acceptance, lane changing);

- the choice of the calibration method (that is, observation of variables at either macro or micro level);

- the dependency of traffic parameters on the characteristics of the specific system to simulate (link, node, network).

Experimental applications have been performed in simulation, and several parameters have been individuated as more significant in term of their effects on fundamental macroscopic variables:

- minimum space distance;

- attributes of vehicles fleet, namely: vehicle length, maximum acceleration, and deceleration;

- driver's sensibility used in the carfollowing model.

The main advantages and warnings for using microscopic simulation models in traffic studies have been discussed.

\section{References}

[1] Smith, M.J.: 'Local traffic control policy which automatically maximizes the overall travel capacity of an urban road network.' Traffic Eng. Control, 1980, 21, (6), pp. 298302.

[2] Taale, H., Van Zuylen, H.J.: 'The Combined Traffic Assignment and Control Problem. An Overview of 25 Years of Research', in 'Proceedings of the 9th World Conference on Transport Research' (2001)

[3] Cipriani, E., Fusco, G.: 'Global optimization of signal settings subject to either system optimal or user equilibrium traffic assignment', in 'Euro Working Group on Transportation' (2002)

[4] Fusco, G., Gori, S.: 'Reti stradali urbane in condizioni dinamiche di sovra-saturazione' (in Rilievi e modellizzazione del traffico veicolare, Seminario, 1993)

[5] Fusco, G., Gentile, G., Meschini, L., et al.: 'Interaction between Signal Settings and Traffic Flow Patterns on Road Networks', in 'Euro Working Group Joint Conference on Transportation' (2006)

[6] Colombaroni, C., Domenichini, L., Salerno, G. (2013) The "Hourglass" model/Il modello "Clessidra" Ingegneria Ferroviaria Volume 68, Issue 7-8, July 2013, Pages 651-660

[7] Torrisi, V., Ignaccolo, M., Inturri, G. (2017). Analysis of road urban transport network capacity through a dynamic assignment model: validation of different measurement 
methods. Transportation Research Procedia, 27, 1026-1033.

[8] Shepelev, V., Aliukov, S., Nikolskaya, K., Shabiev, S. (2020). The Capacity of the Road Network: Data Collection and Statistical Analysis of Traffic Characteristics. Energies, 13(7), 1765.-168047.

[9] Shi, J., Chen, L., Qiao, F., Yu, L., Li, Q., Fan, G. (2020). Simulation and analysis of the carrying capacity for road networks using a grid-based approach. Journal of Traffic and Transportation Engineering (English Edition), 7(4), 498-506.

[10] Yang, H., Bell, M. G., Meng, Q. (2000). Modeling the capacity and level of service of urban transportation networks. Transportation Research Part B: Methodological, 34(4), 255275.

[11] Zhang, P., Yue, H., Zhang, X., Shao, C., Gao, W. (2019). Modeling the Equilibrium Road Network Capacity. IEEE Access, 7, 168029.

[12] Caliper: 'Transmodeler User's Guide, Version $1.5^{\prime}$ (2007)

[13] Gazis, D.C., Herman, R.L, Rothery, R. W. (1961). Non linear follow the leader models of traffic flow. Op. Res., 9(4), pp. 545-567

[14] Rothery, R.: '4. Car Following Models', in 'TRB Special Report 165 - Revised Monograph on Traffic Flow Theory' (2001), pp. 4-1, 4-42

[15] Greenberg, H.: 'An Analysis of Traffic Flow'Oper. Res., 1959, 7, (1), pp. 79-85.

[16] Louah, G.: 'Priority Intersection: Modelling', in Papageorgiou, M. (Ed.): 'Concise Encyclopedia of Traffic \& Transportation Systems' (1991)

[17] Troutbeck, R.J., Brilon, W.: 'Unsignalized Intersection Theory"' (Gartner, 1998)

[18] Petruccelli, U.: 'Il deflusso delle correnti principali nelle intersezioni regolate a precedenza' (Franco Angeli, 1998)

[19] Ancora, V., Fusco, G.: 'A Logit-Based MicrosimualtionModel of Unsignalized Intersections', in 'Euro Working Group on Transportation' (2000)

[20] Smeed, R.J.: 'The road capacity of city centers'Highw. Res. Rec., 1967, 169, (1), pp. 22-29.

[21] Mahmassani, H.S., Williams, J.C., Herman, R.: 'Performance of urban traffic networks'Proc. 10th Int. Symp. Transp. Traffic Theory, 1987, (January 1987), pp. 120.

[22] 'Dynasmart-P Version 1.0: User's Guide' (2004)
[23] Jayakrishnan, R., Mahmassani, H.S., Hu, T.Y.: 'An evaluation tool for advanced traffic information and management systems in urban networks' Transp. Res. Part C, 1994, 2 , (3), pp. 129-147.

[24] 'Dynameq user's manual - Release 1.1.' (2005)

[25] C. Colombaroni, G. Fusco (2015) "Artificial Neural Network Models For Car Following: Experimental Analysis And Calibration Issues. Journal Of Intelligent Transportation Systems", ISSN: 1547-2450

[26] G. Fusco, C. Colombaroni, A. Gemma, S. Lo Sardo (2013). A Quasi-Dynamic Traffic Assignment Model for Large Congested Urban Road Networks. International Journal of Mathematical Models and Methods in Applied Sciences, 7 (4), pp. 341-349. ISSN: 1998-0140.

[27] Isaenko, N.,Colombaroni, C., Fusco, G. (2017) "Traffic dynamics estimation by using raw floating car data" 5th IEEE International Conference on Models and Technologies for Intelligent Transportation Systems, MT-ITS 2017 - Proceedings 8005604, pp 704-709.

[28] De Felice, M., Baiocchi, A., Cuomo, F., Fusco, G., \& Colombaroni, C. (2014). Traffic monitoring and incident detection through VANETs. In 2014 11th Annual Conference on Wireless On-demand Network Systems and Services (WONS), pp.122-129.

\section{Creative Commons Attribution License 4.0 (Attribution 4.0 International, CC BY 4.0)}

This article is published under the terms of the Creative Commons Attribution License 4.0

https://creativecommons.org/licenses/by/4.0/deed.en US 